\title{
Association of Sonographic Features of Unifocal Papillary Thyroid Carcinoma With Cervical Lymph Node Metastasis
}

\section{Liuhua Zhou}

Zhejiang Chinese Medical University

\section{Qiaodan Zhu}

Zhejiang Chinese Medical University

Jincao Yao

Zhejiang Cancer Hospital

Chen Yang

Zhejiang Cancer Hospital

Dong Xu ( $\nabla$ xudong@zjcc.org.cn )

Zhejiang Cancer Hospital

\section{Research}

Keywords: Ultrasonography, Thyroid Cancer, Papillary, Lymphatic metastasis

Posted Date: August 27th, 2021

DOI: https://doi.org/10.21203/rs.3.rs-839643/v1

License: (c) (i) This work is licensed under a Creative Commons Attribution 4.0 International License. Read Full License 


\section{Abstract}

Background Papillary thyroid carcinoma (PTC) is the most common pathological type of thyroid carcinoma. We aim to evaluate the association of sonographic features of PTC and cervical lymph node metastasis (CLNM) at the initial surgery.

Methods Clinical information, ultrasonographic measurements and features for 1335 patients were acquired in data collection. Univariate analysis was performed to test CLNM by 7 independent variables. Receiver operating characteristic (ROC) curve was created to evaluate the diagnostic performance.

Results Univariate analysis showed that gland, location, aspect ratio, margin and echogenic foci were independently associated with CLNM metastatic status $(P<0.05)$. Binary linear regression analysis showed that sex, age, tumour maximum diameter and volume, location, margin and echogenic foci were independent correlative factors. The ROC curves were established based on the relevant factors, the AUC of tumour maximum diameter, tumour volume and margin were $0.74,0.73$, and 0.71 , respectively. The multiple-variable linear regression model was constructed with AUC of 0.81 , specificity of $72.8 \%$, and sensitivity of $75.0 \%$. ANOVA variance analysis for sub-positive groups, tumour maximum diameter, tumour volume, margin and echogenic foci had statistical significance $(P<0.05)$.

Conclusion Younger age, male, larger tumour, margin, and echogenic foci were high risk factors for CLNM in PTC. Cross-sectional aspect ratio with value $\geq 1$ had higher predictive value for CLNM in PTC patients excluding papillary thyroid microcarcinoma (PTMC).

\section{Key Points}

Papillary thyroid carcinoma (PTC) is prone to cervical lymph node metastasis (CLNM), but ultrasonography cannot detect it well.

We use cohort study for 1335 patients, and find out that the location and size of the lesion, margin and punctate echogenic foci are factors with high risk for CLNM. Cross-sectional aspect ratio with value $\geq 1$ had higher predictive value for CLNM in PTC patients excluding papillary thyroid microcarcinoma (PTMC).

Nodular sonographic features are useful clues for routine thyroid scanning and can be utilized to improve the detection rate of CLNM.

\section{Background}

As a noninvasive imaging examination method, ultrasonography has been widely used in clinic, since the ultrasonographic images of the benign and malignant thyroid nodules overlap to some extent, TI-RADS regulates the classification diagnostic criteria, which has a better guidance in judgement of thyroid nodules. The TI-RADS white paper which was proposed by American College of Radiology (ACR) in 2017 
has been internationally recognized and used recently [1], it presented the characteristics of thyroid nodules based on sonographic features such as composition, echogenicity, shape, margin and echogenic foci.

PTC is the most common pathological type of thyroid carcinoma, among which unifocal tumour often occurs and the incidence of multifocal is about 30\% [2]. CLNM is prone to occur early, and there is a risk of postoperative recurrence and distant metastasis [3]. However, there are many limitations in ultrasonography of cervical lymph nodes, especially central lymph nodes metastasis, ultrasonography has the detection rate of $18.8 \%-31 \%$, it is limited because of the interference from trachea, esophagus, osseous tissue, thyroid underlying diseases and the examiner's experience [4-5]. In recent years, preventive central lymph node dissection is executed for PTC patient [6-8], but according to 2015 American Thyroid Association (ATA) Management Guidelines [9], thyroidectomy without prophylactic central neck dissection is appropriate for small ( $\mathrm{T} 1$ or $\mathrm{T} 2$ ), noninvasive, clinically node-negative PTC (cNO). We hope high risk factors of PTC nodules with CLNM could be stratified in routine ultrasound examination, they can help head \& neck surgeons and radiologists assess and complete preoperative surgical planning, then prophylactic neck dissection (central/lateral) will be offered accordingly. It is useful to improve treatment protocol and prognosis.

Ultrasonography is the primary inspection method for thyroid gland, nodular sonographic features are easy to access according to the basic training. In this study, unifocal PTC was selected to analyze in combination with clinical information, ultrasonographic features and ACR ti-radS. It is necessary for us to find some clues to predict the risk of lymph node metastasis before surgery when we perform routine thyroid and cervical lymph node ultrasonic screening.

\section{Materials And Methods}

\section{Patients}

The participants were symptomatic with palpable or incidental thyroid lumps, and all finally underwent thyroidectomy in Cancer Hospital of the University of Chinese Academy of Sciences (Zhejiang Cancer Hospital) from July 2014 to September 2018 were enrolled. Inclusion criteria: 1. thyroidectomy performed for the first time; 2 . enhanced CT scan of neck and thorax to assess cervical lymph node and pulmonary metastasis, and ultrasonography performed for thyroid and neck before operation; 3. PTC was confirmed by biopsy before surgery, all histologic types of PTC including classic and variant were enrolled in the study. Exclusion criteria: 1. lung or other distant metastasis; 2. postoperative pathology diagnosed multifocal PTC. Unilateral thyroid lobe plus isthmus excision or total thyroidectomy were performed for the patients. If lateral lymph node metastasis were suspected by preoperative comprehensive evaluation, and confirmed by biopsy, lateral lymph node dissection would be performed [6-7]. All patients underwent preventive central lymph node dissection [6-8]. whether the dissected lymph node metastasized or not, metastatic lymph nodes were confirmed by pathology. This study was approved by the Ethics Committee 
of Cancer Hospital of the University of Chinese Academy of Sciences (Zhejiang Cancer Hospital). Informed consent was obtained from all enrolled patients to include the data for analysis.

\section{Instruments}

GE Logiq E9 ultrasonographic instrument (General Electric Healthcare, Milwaukee, WI, USA) with a highresolution linear probe (ML6-15) and Philips iU22 ultrasonographic instrument (Royal Dutch Philips Electronics, Amsterdam, Noord-Holland, Netherlands) with a high frequency linear probe (L12-5) were used for the examination.

\section{Protocol}

Patients were maintained at supine position with neck hyper-extended, then thyroid and double-sides neck were scanned at multi-section. The three diameters (length, width and depth), lesion location, composition, echogenicity, shape, margin and echogenic foci of the tumour were recorded and evaluated from workstations. The ultrasonographic images were performed by two same professional physicians with experience of more than 10 years, both of them were board-certified physicians with training and experience of thyroid US. The ultrasonographic images and reports were analyzed in a blind manner by another two ultrasound specialists (with experience of more than 10 years) independently. All the imaging data were compared to the pathological results from neck dissections. For measurements, the error was less than $2 \mathrm{~mm}$ for two ultrasound specialists, average value was applied. When the measurement error exceeded $2 \mathrm{~mm}$, and any discordance for ultrasonographic features occurred, the completely separate experienced sonologists (with experience of more than 20 years) in thyroid US reviewed the images and the final decision was determined.

Clinical information, ultrasonographic measurements and features were used for data collection. Clinical information included sex, age; ultrasonographic measurements included the tumour maximum diameter, tumour volume $(\mathrm{V}=0.523 *$ length *width*depth), cross-sectional and longitudinal aspect ratio; ultrasonographic features included ACR TI-RADS, gland represented left/right/isthmus, location represented upper and lower (longitudinal scan)/anterior and posterior (longitudinal scan)/inside and outside (transversal scan) for each lobe. The three diameters of the tumour were stated precisely as the following, we did a longitudinal scan of thyroid, selected the maximum section of the nodule, measured the maximum long diameter, it was length, then measured vertical diameter of long diameter, it was depth. Transversal scan of thyroid was executed, selected the maximum section of the nodule, measured the maximum diameter from left to right, it was width.

Cross-sectional aspect ratio=depth/width and longitudinal aspect ratio=depth/length. Sonographic features according to ACR TI-RADS were carried out: composition (cystic, mixed cystic and solid, solid or almost completely solid), echogenicity (anechoic, hyperechoic or isoechoic, hypoechoic), shape (aspect ratio<1or $\geq 1$ ), margin (smooth or ill-defined/lobulated or irregular/extra-thyroid extension) and echogenic foci (none or large comet-tail artifacts/ macro/ peripheral / punctate echogenic foci). 
TI-RADS score was acquired from the sonographic features, according to the setting of ACR. TI-RADS grade was clarified as the following: TR1=0 Points, TR2=2 Points, TR3=3 Points, TR4=4-6 Points, TR5=7 Points or More.

\section{Statistical analysis}

Patients were divided into two groups according to the presence or absence of CLNM. The obtained data were statistically analyzed by SPSS 20.0 software. Continuous quantitative data were expressed as the mean \pm standard deviation (SD). Data-counting were described statistically by the number of cases and rates. In this study, total four steps for statistical analysis were adopted.

Step1, we used Chi-square test and independent-sample T test to compare the categorical variables of sonographic features, they were univariate analysis and 2-sided hypothesis tests. Step2, a forward binary linear regression analysis was adopted with clinical information, ultrasonographic measurements and the categorical variables if analysis index $\mathrm{P}<0.05$ in the univariate analysis to further determine whether it was an independent correlative factor for CLNM.

Step3, Odds ratios (ORs) with 95\% confidence intervals (Cls) were calculated and receiver operating characteristic curves (ROC) were analyzed for factors with significance on binary linear regression analysis. The corresponding area under the curve (AUC) and sensitivity and specificity of each significant independent variable and binary linear regression analysis fitting equation were used to predict the risk of CLNM. Multiple linear regression equation reflects the relationship between independent variables and covariates, covariates are originated from binary linear regression analysis which conform to $P<0.05$.

Step4, the positive group were divided into three groups, including only central lymph node metastasis group, only lateral lymph node metastasis group and both metastatic group. ANOVA variance analysis was used between the three groups for pair-to-pair comparison in order to find out the differences in clinical information, ultrasonographic measurements and features. The potential errors due to multiple comparisons for secondary and sub-group were handled by adjusting significance threshold. $\mathrm{P}<0.05$ was considered statistically significant.

\section{Results}

The appropriate patients were selected retrospectively according to the inclusion and exclusion criteria, a total of 1335 participants were enrolled with intact imaging data for the accurate assessment of PTC. There were 299 males and 1036 females, withath mean age 45.3 years (range 12 to 84 years); the average tumour maximum diameter was $10.3 \mathrm{~mm}$ (range 3.5 to $64.7 \mathrm{~mm}$ ). There were 874 cases of PTMC and 461 cases of PTC patients excluding PTMC among all. 432 cases were in positive group (32.4\%), PTMC patients were 181 cases, PTC excluding PTMC were 251 cases. Among the positive group, $285(21.4 \%)$ with only central lymph node metastasis, 54 (4.0\%) with only lateral lymph node metastasis, and $93(7.0 \%)$ with metastasis both in central and lateral lymph node) were included. There were 903 cases included in negative group (67.6\%), PTMC patients were 693 cases, PTC excluding PTMC were 210 
cases. 143 patients underwent lateral lymph node dissection due to the positive pre-operative biopsy. Lateral neck lymph node dissection was performed in 5 cases with high imaging suspicion but negative puncture results. 3 cases of them had lateral neck lymph node metastasis and 2 cases were confirmed negative during operation.

All cervical lymph node metastases were found out at the time of initial surgery, recurrent disease in cervical lymph nodes were not included. By comparing ultrasonographic features of patients in positive and negative group with Chi-square test and independent-sample $T$ test, the results showed that gland, location, aspect ratio, margin and echogenic foci showed significant differences (Table 1). 
Table 1

Univariate analysis of PTC with CLNM

\begin{tabular}{|c|c|c|c|c|}
\hline \multicolumn{2}{|l|}{ Variable } & \multirow[t]{2}{*}{$\begin{array}{l}\text { Positive group }(n= \\
432)\end{array}$} & \multirow[t]{2}{*}{$\begin{array}{l}\text { Negative group }(n= \\
903)\end{array}$} & \multirow{2}{*}{$\begin{array}{l}P \\
P= \\
0.001\end{array}$} \\
\hline Gland & & & & \\
\hline & Left & $177(41.0)$ & $414(45.8)$ & \\
\hline & Right & 213(49.3) & $449(49.7)$ & \\
\hline & Isthmus & $42(9.7)$ & $40(4.5)$ & \\
\hline Location & & & & $\begin{array}{l}P= \\
0.002\end{array}$ \\
\hline & Upper anterior inside & $119(27.6)$ & 283(31.3) & \\
\hline & Upper anterior outside & $101(23.4)$ & $185(20.5)$ & \\
\hline & Upper posterior inside & $42(9.7)$ & 119(13.2) & \\
\hline & Upper posterior outside & $28(6.5)$ & $59(6.5)$ & \\
\hline & Lower anterior inside & $23(5.3)$ & $62(6.9)$ & \\
\hline & Lower anterior outside & $28(6.5)$ & $36(4.0)$ & \\
\hline & Lower posterior inside & $23(5.3)$ & $61(6.8)$ & \\
\hline & Lower posterior outside & $26(6.0)$ & $58(6.4)$ & \\
\hline & Isthmus & $42(9.7)$ & $40(4.4)$ & \\
\hline Composition & & & & $\begin{array}{l}P= \\
0.09\end{array}$ \\
\hline & mixed cystic and solid & $10(2.3)$ & 10(1.1) & \\
\hline & $\begin{array}{l}\text { solid or almost completely } \\
\text { solid }\end{array}$ & 422(97.7) & 893(98.9) & \\
\hline Echogenicity & & & & $\begin{array}{l}P= \\
0.19\end{array}$ \\
\hline & hypoechoic & 424(98.1) & 894(99.0) & \\
\hline & hyperechoic or isoechoic & $8(1.9)$ & $9(1.0)$ & \\
\hline Aspect ratio & & & & $\begin{array}{l}P< \\
0.001\end{array}$ \\
\hline & $<1$ & $214(49.5)$ & $322(35.7)$ & \\
\hline & $\geq 1$ & $218(50.5)$ & $581(64.3)$ & \\
\hline
\end{tabular}




\begin{tabular}{|c|c|c|c|c|}
\hline Variable & & $\begin{array}{l}\text { Positive group }(n= \\
\text { 432) }\end{array}$ & $\begin{array}{l}\text { Negative group }(n= \\
903)\end{array}$ & $\mathbf{P}$ \\
\hline \multirow[t]{4}{*}{ Margin } & & & & $\begin{array}{l}P< \\
0.001\end{array}$ \\
\hline & smooth or ill-defined & $171(39.6)$ & 732(81.1) & \\
\hline & lobulated or irregular & $229(53.0)$ & $160(17.7)$ & \\
\hline & extra-thyroid extension & $32(7.4)$ & $11(1.2)$ & \\
\hline \multirow[t]{5}{*}{$\begin{array}{l}\text { Echogenic } \\
\text { foci }\end{array}$} & & & & $\begin{array}{l}P< \\
0.001\end{array}$ \\
\hline & $\begin{array}{l}\text { none or large comet-tail } \\
\text { artifacts }\end{array}$ & $174(40.3)$ & $664(73.5)$ & \\
\hline & macro & $8(1.8)$ & $21(2.3)$ & \\
\hline & peripheral & $38(8.8)$ & $32(3.6)$ & \\
\hline & punctate echogenic foci & 212(49.1) & 186(20.6) & \\
\hline
\end{tabular}

We divided two sub-groups in positive group, there were PTMC and PTC excluding PTMC. Compared these two groups, cross-sectional aspect ratio $\geq 1$ had significant difference for PTC patients excluding PTMC to predict CLNM (Table 2).

Table 2

Univariate analysis of PTMC and PTC excluding PTMC in the positive group

\begin{tabular}{|llll|}
\hline Variable & $\begin{array}{l}\text { Cross- } \\
\text { sectional } \\
\text { aspect ratio } \geq\end{array}$ & $\begin{array}{l}\text { longitudinal section aspect ratio } \\
\mathbf{1}\end{array}$ & $P$ \\
\hline PTMC + CLNM (181) & 118 & 93 & $P=0.006$ \\
\hline $\begin{array}{l}\text { PTC excluding PTMC + CLNM } \\
(251)\end{array}$ & 80 & 32 & \\
\hline
\end{tabular}

The clinical information, ultrasonographic measurements and features were included in the binary linear regression analysis. Aspect ratio was included in cross-sectional and longitudinal section aspect ratio. The result showed that sex, age, maximum tumour diameter and volume, location, margin and echogenic foci were independent risk factors for CLNM (Table 3). 
Table 3

Binary logistic regression analysis of PTC with CLNM

\begin{tabular}{|llll|}
\hline Variable & OR & $95 \%$ Cls & $\mathrm{P}$ \\
\hline Overall $(\mathrm{N}=432, \mathrm{n}=903)$ & & & \\
\hline Sex & 1.75 & $1.28 \sim 2.40$ & $\mathrm{P}=0.001$ \\
\hline Age & 0.96 & $0.95 \sim 0.97$ & $\mathrm{P}<0.001$ \\
\hline Maximum tumour diameter & 1.09 & $1.05 \sim 1.13$ & $\mathrm{P}<0.001$ \\
\hline Tumour volume & 0.92 & $0.86 \sim 0.99$ & $\mathrm{P}=0.03$ \\
\hline Cross-sectional aspect ratio & 1.92 & $0.87 \sim 4.26$ & $\mathrm{P}=0.11$ \\
\hline Longitudinal section aspect ratio & 1.33 & $0.73 \sim 2.47$ & $\mathrm{P}=0.38$ \\
\hline Gland (left/right/ isthmus) & 1.15 & $0.90 \sim 1.47$ & $\mathrm{P}=0.25$ \\
\hline Location & 1.07 & $1.01 \sim 1.13$ & $\mathrm{P}=0.02$ \\
\hline Margin & 2.04 & $1.76 \sim 2.37$ & $\mathrm{P}<0.001$ \\
\hline Echogenic foci & 1.27 & $1.14 \sim 1.42$ & $\mathrm{P}<0.001$ \\
\hline N The number of patients for positive group; $\mathrm{n}$ The number of patients for negative group \\
\hline
\end{tabular}

Binary linear regression analysis and the ROC curve were used to obtain the independent correlative factors for CLNM. The AUC, specificity, sensitivity of maximum tumour diameter was $0.74,66.6 \%$, and $69.9 \%$, respectively. For tumour volume, the AUC, specificity and sensitivity were $0.73,67.6 \%$, and $68.1 \%$, respectively. For margin, the AUC, specificity and sensitivity were $0.71,81.1 \%$, and $60.4 \%$, respectively. To take CLNM in PTC patients as a dependent variable, independent risk factors as covariates, a regression equation of CLNM was deduced from the parameters, $Y=-2.633+0.561 * X 10.043 * X 2+0.086 * X 30.083 * X 4$ $+0.066 * X 5+0.703 * X 6+0.219 * X 7$ ( $Y$ represented as $C L N M, X 1=$ sex, $X 2=$ age, $X 3=$ maximum tumour diameter, $\mathrm{X} 4$ = tumour volume, $\mathrm{X} 5$ = location, $\mathrm{X} 6$ = margin, $\mathrm{X} 7$ = echogenic foci). The ROC curve was used to evaluate the regression model constructed by combining seven independent correlative factors (sex, age, maximum tumour diameter and volume, location, margin and echogenic foci), in which the value of AUC, specificity and sensitivity was $0.81,72.8 \%$ and $75.0 \%$, respectively (Table 4 ). 
Table 4

ROC analysis of the independent variables in identifying PTC with CLNM

\begin{tabular}{|lllll|}
\hline Variable & AUC & $95 \%$ Cls & Specificity & Sensitivity \\
\hline Overall $(\mathrm{N}=432, \mathrm{n}=903)$ & & & & \\
\hline Sex & 0.55 & $0.52 \sim 0.59$ & $81.0 \%$ & $29.4 \%$ \\
\hline Age & 0.36 & $0.32 \sim 0.39$ & $99.9 \%$ & $0.2 \%$ \\
\hline Tumor maximum diameter & 0.74 & $0.71 \sim 0.77$ & $66.6 \%$ & $69.9 \%$ \\
\hline Tumor volume & 0.73 & $0.71 \sim 0.76$ & $67.6 \%$ & $68.1 \%$ \\
\hline Location & 0.53 & $0.50 \sim 0.56$ & $95.6 \%$ & $9.7 \%$ \\
\hline Margin & 0.71 & $0.68 \sim 0.74$ & $81.1 \%$ & $60.4 \%$ \\
\hline Echogenic foci & 0.67 & $0.64 \sim 0.70$ & $75.9 \%$ & $57.9 \%$ \\
\hline Equation & 0.81 & $0.79 \sim 0.84$ & $72.8 \%$ & $75.0 \%$ \\
\hline N The number of patients for positive group; $\mathrm{n}$ The number of patients for negative group \\
\hline
\end{tabular}

According to the differences in clinical information, ultrasonographic measurements and features by ANOVA variance analysis, the patients were divided into three sub-positive groups: only central lymph node metastasis group, only lateral lymph node metastasis group and both metastatic group. The results showed that significant variables $(P<0.05)$ between only central and only lateral lymph node metastasis group, between only central and both metastatic group were same, including tumour maximum diameter, tumour volume, margin and Echogenic foci (Table 5).

Table 5

ANOVA variance analysis for CLNM in PTC

\begin{tabular}{|llll|}
\hline Variable & Central vs lateral & Central vs both & Lateral vs both \\
\hline Gender(male/female) & $\mathrm{P}=0.993$ & $\mathrm{P}=0.155$ & $\mathrm{P}=0.324$ \\
\hline Age & $\mathrm{P}=0.476$ & $\mathrm{P}=0.493$ & $\mathrm{P}=0.147$ \\
\hline Tumor maximum diameter & $\mathrm{P}<0.001^{\mathrm{a}}$ & $\mathrm{P}<0.001^{\mathrm{a}}$ & $\mathrm{P}=0.02^{\mathrm{a}}$ \\
\hline Tumor volume & $\mathrm{P}<0.001^{\mathrm{a}}$ & $\mathrm{P}<0.001^{\mathrm{a}}$ & $\mathrm{P}=0.322$ \\
\hline Location & $\mathrm{P}=0.779$ & $\mathrm{P}=0.766$ & $\mathrm{P}=0.056$ \\
\hline Margin & $\mathrm{P}<0.001^{\mathrm{a}}$ & $\mathrm{P}<0.001^{\mathrm{a}}$ & $\mathrm{P}=0.869$ \\
\hline Echogenic foci & $\mathrm{P}<0.001^{\mathrm{a}}$ & $\mathrm{P}<0.001^{\mathrm{a}}$ & $\mathrm{P}=0.945$ \\
\hline a represented $\mathrm{P}<0.05$ & & & \\
\hline
\end{tabular}




\section{Discussion}

With the application of high frequency ultrasound, the incidence of thyroid lesions in adults can be up to $60 \sim 70 \%$ [10]. Fine-needle aspiration (FNA) has been the most effective diagnostic method for benign and malignant thyroid neoplasms recently [11]. However, regular observation is one of the choices for most benign nodules, even malignant nodules, especially malignant nodules $<1 \mathrm{~cm}$ or selected patients with contraindications for surgical procedure [12-13]. Therefore, the standardization of thyroid ultrasonographic images by ti-rads can effectively improve the differential diagnosis of thyroid nodules.

PTC is the most common malignant thyroid tumour, lymph node metastasis of which were associated with the diameter, location, number and invasive growth of primary tumour [14-15]. The sensitivity of ultrasound for the evaluation of central and lateral compartment lymph nodes is around $60 \%$ [9], in this study, compared with cN1 and pN1, the detection rate of cervical lymph node metastases was $64.8 \%$ (280/432). CLNM in PTC generally first occurs in the central region and then to the lateral region [16], which is more common in area VI, followed by area III and IV [17]. However, not all PTC lymph node metastases follow this pattern, with some skip metastasis where lymph node metastasis occurs in the lateral neck region and no metastasis in the ipsilateral central region [18]. Some studies have reported that the rate of lymph node skip metastasis was $3 \sim 21.8 \%$ [19]. Due to only unifocal PTC included in this study, the lymph node skip metastasis rate was $4.0 \%$ (54/432).

TI-RADS was mainly adopted in the differential diagnosis of benign and malignant thyroid neoplasms. The consistent conclusion of the association between sex, age and sonographic features and lymph node metastasis was difficult to achieve [20-22]. This study only included unifocal PTC and conducted a stratified study to comprehensively evaluate the risks of CLNM in PTC combined with clinical information, ultrasonographic measurements and TI-RADS to obtain a more complete and effective result.

PTC can occur in any part of thyroid, including bilateral lobes and isthmus. For isthmus, the incidence of thyroid cancer is about 2.5\% 9.2\% [23]. Studies have found that isthmus PTC was more likely to invade thyroid capsule and surrounding tissues, compared with the lateral lobe PTC [24-25]. This is mainly because the area of the isthmus tumour in contact with thyroid capsule is relatively large, which is easy to invade the capsule or break through the capsule and invade the surrounding tissues, thus CLNM would happen. Previous studies [25-26] have suggested that when the tumour was located in the middle or lower pole of thyroid, the risk of CLNM was increased. In this study, the incidence of PTC lymph node metastasis in isthmus was larger than in the left and right lobes $(51.2 \%(42 / 82)$ vs $29.9 \% \bigotimes 177 / 591$ 区vs $32.2 \% \varangle 213 / 662 \rrbracket)$, and the lobes were stereolocated by the upper and lower, anterior and posterior, inside and outside, $43.8 \%$ (28/64) of lymph node metastasis occurred in the lower, anterior and outside location, which were consistent with previous studies [25-26]. We recommend principle of stereo-position for lesions, in one hand, we can assess the clearer location of the lesion, in other hand, for isthmus and lower lesion, we should check the neck carefully for CLNM. However, more data should be provided to promote and confirm the result. 
Aspect ratio $\geq 1$ is a highly specific index for the diagnosis of malignant thyroid nodules [27-28]. According to previous literature, the results of association between aspect ratio and CLNM in PTC were not consistent. Part of literature [29-30] suggested that it was prone to occur CLNM when aspect ratio > 1 , and CLNM was the risk factor. The study [31] reported that no statistical significance could be seen in the prediction of lateral cervical lymph node metastasis in the case of aspect ratio $\geq 1$. In this study, aspect ratio had statistical significance in univariate analysis, while we used the binary logistical analysis, there was no significant difference, the main reason was that PTMC patients were $65.5 \%$ (874/1335) in the study, just $20.7 \%$ (181/874) were in the positive group. In addition, according to the morphology of the thyroid lesion, it could be divided into cross-sectional aspect ratio and longitudinal section aspect ratio. We compared cross-sectional aspect ratio and longitudinal section aspect ratio for PTMC and PTC patients excluding PTMC, it could be concluded that cross-sectional aspect ratio had a better predictive value for CLNM in PTC excluding PTMC, compared with the longitudinal aspect ratio. There was less relevant literature with the association between cross-sectional and longitudinal section aspect ratios in predicting cervical LN metastasis, especially for PTC patients excluding PTMC. We will collect more data for further research in the future.

Malignant tumour grows rapidly, the cancer cells continue to invade outward, then the incidence of lymph node metastasis is relatively increased [32-33]. Margin is one of the invasive characteristics of the tumour. The nodules with high invasiveness showed irregular and lobulated boundaries, while smooth boundaries generally indicate low invasiveness and slow growth [34]. In this study, univariate analysis and logistic regression analysis both showed a good association between margin and CLNM in PTC, the lesions with lobular/irregular or extension more occurred CLNM than smooth or ill-defined $(60.4 \%$ $(261 / 432)$ vs $39.6 \%(171 / 432))$. This is consistent with the results reported in the literature $[21,26]$. Margin had statistical significance $(P<0.001)$ between only central lymph node metastasis group and only lateral lymph node metastasis group, between only central lymph node metastasis group and both metastatic group by ANOVA variance analysis.

Echogenic foci are divided into micro-calcification, macro-calcification and ring calcification around the nodules on the basis of $1 \mathrm{~mm}$ [35]. Micro-calcification can reflect the psammoma bodies in pathology, which results from calcification and necrosis of cancer cells and is a specific indicator for the diagnosis of PTC [36], and it is significantly related to lymph node metastasis. Continuous follow-up studies found that CLNM was more likely to occur in PTC with micro-calcification [37]. In this study, the patients with peripheral calcification or punctate echogenic foci had more CLNM, compared with none or large comettail artifacts $(57.9 \%(250 / 432)$ vs $42.1 \%(182 / 432))$. When ANOVA variance analysis was executed, echogenic foci also had significant difference $(P<0.001)$. Therefore, ultrasonography can better predict the risk of CLNM in PTC for the different types of calcifications.

Ti-rads comprehensively evaluated the tumour according to sonographic features of thyroid nodules. Its scoring and grading system were used for the differential diagnosis of benign and malignant nodules, it would be great value for further determination of diagnosis and treatment protocols. In this study, sex, age, ultrasonographic measurements and features were included for logistic regression analysis to 
establish a prediction model for CLNM, with a specificity of $72.8 \%$ and a sensitivity of $75.0 \%$. Sun et al. [38] reported that they used sex, age, max diameter and number of nodules, cervical lymph node detected by ultrasound as covariates, CLNM as a dependent variable, a prediction model was acquired with a specificity of $80.8 \%$ and a sensitivity of $59.8 \%$. The result had reference value. It also demonstrates the importance of comprehensive evaluation of ultrasound in clinical practice.

Heaton et al. [39] reported that women and elderly patients were at risk of PTC, while men and younger patients were at risk of CLNM. In this study, CLNM occurred in $42.5 \%(127 / 299)$ of male patients, and occurred in only $29.4 \%(305 / 1036)$ of female patients, which also suggests that male patients have a higher risk of lymph node metastasis. We used 55 years as the threshold in this study according to the TNM staging system for thyroid cancer [40], and 35.4\% (371/1048) of patients who were younger than 55 years had CLNM, and among those who were 55 years or older, lymph node metastases occurred in only $21.2 \%(61 / 287)$ of patients in this study.

The maximum tumor diameter is an important reference index for PTC treatment protocols and the range of surgery [41]. The results demonstrated that the average maximum tumor diameter in the positive group was about 1.7 times $(14.4 \mathrm{~mm}$ vs $8.36 \mathrm{~mm})$ that in the negative group. The tumor seemed to be ellipsoid, and the volume as the evaluation parameter made the result more objective and scientific. The average tumor volume in the positive group was about 3.7 times $(2.05 \mathrm{ml} v \mathbf{v s} 0.55 \mathrm{ml})$ that in the negative group. For larger tumors, cervical lymph nodes should be examined in order to improve the detection rate of CLNM. In particular, in patients with large tumors, central or both central and lateral lymph node metastasis should be determined in advance.

Limitations of this study: 1. It was a single-center retrospective study including unifocal PTC and lymph node dissection performed in the central area, which may cause selection bias. 2. Cases with metastases in lateral location were not adequate, large samples are required to study the cervical metastases in different parts. For skip lateral lymph node metastases, more effective preoperative assessment should be adopted. 3. This is our preliminary study for large sample size of PTC patients, we will add detailed clinical and pathological staging, subdivided pathological types, machine learning models for further research.

\section{Conclusions}

In conclusion, based on PTC pathology, the correlations between sex, age, ultrasonographic measurements and features were analyzed in order to assess a good clinical value for preoperative evaluation of the risk of lymph node metastasis. For PTC patients with high-risk factors such as less than 55 years, male, larger tumour, lobulated or irregular margin, peripheral calcification or punctate echogenic foci, preoperative lymph node examination should be conducted in details, including cervical lymph node sonographic scan by the experienced sonologists (with experience of more than 10 years) and enhanced CT scan of neck as a rule, which will cover a wide range of thyroid cancer population and help develop a more reasonable clinical treatment protocol. 


\section{Abbreviations}

PTC Papillary thyroid carcinoma

CLNM Cervical lymph node metastasis

PTMC Papillary thyroid microcarcinoma

TI-RADS Thyroid imaging report and data system

ACR American College of Radiology

SD Standard Deviation

OR Odds ratio

$\mathrm{Cl}$ Confidence interval

ROC Receiver operating characteristic

AUC Area under the curve

FNA Fine-needle aspiration

\section{Declarations}

\section{Ethics approval and consent to participate}

This study was approved by the Ethics Committee of Cancer Hospital of the University of Chinese Academy of Sciences (Zhejiang Cancer Hospital). Informed consent was obtained from all enrolled patients to include the data for analysis.

\section{Consent for publication}

The scientific guarantor of this publication is Prof. Dong Xu. The permission has been received that any material in the article can be used, and consent for publication is acquired from all authors.

\section{Availability of data and materials}

The datasets used and analysed during the study are available from the corresponding author on reasonable request.

\section{Competing interests}

We define that all authors haven't involved in a set of conditions in which our professional judgment concerning the validity of research, and we aren't influenced by financial gain. Data and material are 
available and reliable in this article.

\section{Funding}

This study was supported by National Natural Science Foundation of China (NO. 82071946) and Zhejiang Provincial Natural Science Foundation of China (NO. LSD19H180001).

\section{Authors' contributions}

Dong Xu had full access to all the data and took responsibility for the integrity and accuracy of the data analysis. Liuhua Zhou and Jincao Yao were major contributors in writing the manuscript. Qiaodan Zhu participated in collection and management of the data. Chen Yang contributed to analyse and interpret the data. All authors read and approved the final manuscript.

\section{Acknowledgements}

The authors thank Chen Cui PhD for reviewing the manuscript and giving support of data analysis.

\section{References}

1. Franklin NT, Willian D, Edward GG, et al. ACR thyroid imaging, reporting and data system (TI-RADS): white paper of the ACR TI-RADS committee[J]. J Am Coll Radiol. 2017;14:587-95.

2. Lim H, Devesa SS, Sosa JA, et al. Trends in thyroid cancer incidence and mortality in the united states, 1974-2013[J]. JAMA. 2017;317(13):1338-48. DOI:10.1001/jama.2017.2719.

3. Kim SY, Kwak JY, Kim EK, et al. Association of preoperative US features and recurrence in patients with classic papillary thyroid carcinoma[J]. Radiology. 2015;277(2):574-83.

DOI:10.1148/radiol.2015142470.

4. Li JW, Chang $\mathrm{C}$, Chen $\mathrm{M}$, et al. Identification of calcifications in thyroid nodules: comparison between. ultrasonography and CT[J]. Chin J Ultrasonogr. 2017;26(5):3137. DOI:10.3760/cma.j.issn.1004-4477.2017.05.012.

5. Zhu Q, Li JW, Zhou SC, Chang C, Chen M, Fan YW. Diagnostic value of ultrasonography in predicting neck lymph node metastasis in Hashimoto's thyroiditis with papillary thyroid carcinoma[J]. Chin $\mathrm{J}$ Ultrasonogr. 2016;25(11):962-5. DOI:10.3760/cma.j.issn.1004-4477.2016.11.011.

6. Chinese Association of Thyroid Oncology (CATO). Chinese experts' consensus on diagnosis and treatment of thyroid micropapillary carcinoma (2016 Edition). Chin J Clin Oncol. 2016;43(10):40511.

7. Chinese Thyroid Association (CTA), Thyroid Committee of Chinese Research Hospital Association. Expert consensus on lymph node dissection in cervical region of differentiated thyroid cancer (2017 Edition) [J]. Chin J Practical Surg. 2017;37(9):985-91.

8. Wang TS, Evans DB. Commentary on: Occult lymph node metastasis and risk of regional recurrence in papillary thyroid cancer after bilateral prophylactic central neck dissection: A multi-institutional 
study[J]. Surgery. 2017;161(2):472-4.

9. Haugen BR, Alexander EK, Bible KC, Doherty GM, Mandel SJ. 2015 American Thyroid Association Management Guidelines for Adult Patients with Thyroid Nodules and Differentiated Thyroid Cancer. Thyroid.2016; 26(1). DOI:10.1089/thy.2015.0020.

10. Guth S, Theune U, Aberle J, Galach A, Bamberger CM. Very high prevalence of thyroid nodules detected by high frequency $(13 \mathrm{MHz})$ ultrasound examination. Eur J Clin Invest. 2009;39:699-706.

11. Singh Ospina N, Brito JP, Maraka S, et al. Diagnostic accuracy of ultrasound-guided fine needle aspiration biopsy for thyroid malignancy: systematic review and meta-analysis. Endocrine. 2016;53:651-61.

12. Simth-Bindman R, Lebda P, Feldstein VA, et al. Risk of thyroid cancer based on thyroid ultrasound imaging characteristics: results of a population-based study. JAMA Intern Med. 2013;173:1788-96.

13. Davies L, Welch HG. Current thyroid cancer trends in the United States. JAMA Otolaryngol Head Neck Surg. 2014;140:317-22.

14. Haugen BR, Alexander EK, Bible KC, et al. 2015 American Thyroid Association Management Guidelines for Adult Patients with Thyroid Nodules and Differentiated Thyroid Cancer: The American Thyroid Association Guidelines Task Force on Thyroid Nodules and Differentiated Thyroid Cancer[J].2016; 26(1):1-133. DOI:10.1089/thy.2015.0020.

15. Park JP, Roh JL, Lee JH, et al. Risk factors for central neck lymph node metastasis of clinically noninvasive, node-negative papillary thyroid microcarcinoma[J]. Am J Surg. 2014; 208(3):412-418. DOI:10.1016/j. amjsurg. 2013.10.032.

16. Zeng RC, Zhang W, Gao EL, et al. Number of central lymph node metastasis for predicting lateral lymph node metastasis in papillary thyroid microcarcinoma[J]. Head Neck. 2014;36(1):101-6.

17. Kim KE, Kim EK, Yoon JH, Han KH, Moon HJ, Kwak JY. Preoperative prediction of central lymph node metastasis in thyroid papillary microcarcinoma using clinicopathologic and sonographic features[J]. Word J Surg. 2013;37(2):385-91.

18. Lee YS, Shin SC, Lim YS, et al. Tumour location-dependent skip lateral cervical lymph node metastasis in papillary thyroid cancer[J]. Head Nek. 2014;36(6):887-91. DOI:10.1002/hed.23391.

19. Lei J, Zhong J, Jiang K, Li Z, Gong R, Zhu J. Skip lateral lymph node metastasis leaping over the central neck compartment in papillary thyroid carcinoma[J]. Oncotarget. 2017; 8(16): 27022-33. DOI:10.18632/ oncotarget.15388.

20. AL AA, Williams BA, Rigby MH, et al. Multifocal papillary thyroid cancer increases the risk of central lymph node metastasis[J]. Thyroid.2015; 25(9):1008-1012. DOI:10.1089/thy. 2015.0130.

21. Lin DZ, Qu N, Shi RL, Lu ZW, Ji QH, Wu WL. Risk prediction and clinical model building for lymph node metastasis in papillary thyroid microcarcinoma[J]. Onco Targets Ther. 2016;9:530716. DOI:10.2147/OTT.S107913.

22. Qu N, Zhang L, Lin DZ, et al. The impact of coexistent Hashimoto's thyroiditis on lymph node metastasis and prognosis in papillary thyroid microcarcinoma[J]. Tumour Biol. 2016;37(6):7685-92. DOI:10.1007/s13277-015-4534-4. 
23. Lee YS, Jeong JJ, Nam KH, Chung WY, Chang HS, Park CS. Papillary carcinoma located in the thyroid isthmus[J]. World J Surg. 2010;34(1):36-9.

24. Fan FJ, Qin L, Ding HY, Liu DQ, Jia JY, Tan Z. Study on ultrasound image features of papillary thyroid carcinoma originating in the isthmus [J]. Chin J Clinicians (Electronic Edition). 2015;9(20):3720-3.

25. Xiang D, Xie L, Xu Y, Li Z, Hong Y, Wang P. Papillary thyroid microcarcinomas located the middle part of the middle third of the thyroid gland correlates with the presence of neck metastasis[J]. Surgery. 2015; 157(3):526-533. DOI:10.1016/ j. surg. 2014.10.020.

26. Sun $W$, Lan $X$, Zhang $H$, et al. Risk factors for central lymph node metastasis in CNO papillary thyroid carcinoma: a systematic review and meta-analysis[J]. PLoS One. 2015;10(10):e0139021.

DOI:10.1371/ journal. pone. 0139021.

27. Kwak JY, Han $\mathrm{KH}$, Yoon JH, et al. Thyroid imaging reporting and data system for US features of nodules: a step in establishing better stratification of cancer risk. Radiology. 2011;260:892-9.

28. Na DG, Baek JH, Sung JY, et al. Thyroid imaging reporting and data system risk stratification of thyroid nodules: categorization based on solidity and echogenicity. Thyroid. 2016;26:562-72.

29. Nam SY, Shin JH, Han BK, et al. Preoperative ultrasonographic features of papillary thyroid carcinoma predict biological behavior[J]. J Clin Endocrinol Metab. 2013;98(4):1476-82.

30. Zhou J, Zhou SC, Li JW, et al. Risk factors of central neck lymph node metastasis following solitary papillary thyroid carcinoma[J]. Chin J Ultrasonogr. 2019;28(3):235-40.

31. Deng SP, Xiong HH, Li QS, et al. Ultrasonographic characteristics of papillary thyroid cancer: predicting factors of lateral lymph node metastasis[J]. Chinese J Ultrasound Med. 2017;33(3):196-8.

32. Heaton CM, Chang JL, Orloff LA. Prognostic implications of lymph node yield in central and lateral neck dissections for well-differentiated papillary thyroid carcinoma[J]. Thyroid. 2016;26(3):434-40.

33. Lan Y, Song Q, Jin Z, Zhang Y, Lin L, Luo YK. Correction of routine ultrasound features and BRAFV600E gene to the cervical lymph node metastasis of thyroid papillary carcinoma[J]. Med $\mathrm{J}$ Chin PLA. 2019;44(9):747-52.

34. Deng SP, Li QS, Chen SH, et al. Analysis of related factors on cervical lymph node metastasis and ultrasonographic characteristics of papillary thyroid microcarcinoma[J]. J Clin Ultrasound in Med. 2017;19(6):424-6.

35. Oh EM, Chung YS, Song WJ, Lee YD. The pattern and significance of the calcifications of papillary thyroid microcarcinoma presented in preoperative neck ultrasonography[J]. Ann Surg Treat Res. 2014;86(3):115-21.

36. Shi C, Li S, Shi T, Liu B, Ding C, Qin H. Correlation between thyroid nodule calcification morphology on ultrasound and thyroid carcinoma[J]. J Int Med Res. 2012;40(1):350-7.

37. Bai Y, Zhou G, Nakamura M, et al. Survival impact of psammoma body, stromal calcification and bone formation in papillary thyroid carcinoma[J]. Mod Pathol. 2009;22(7):887-94.

38. Sun YS, Lv HJ, Zhao YR, Shi BY. Risk factors for central neck lymph node metastases of papillary thyroid carcinoma[J]. Chin J Otorhinolaryngol Head Nexk Surg. 2017;52(6):421-5. 
39. Heaton CM, Chang JL, Orloff LA. Prognostic implications of lymph node yield in central and lateral neck dissections for well-differentiated papillary thyroid carcinoma[J]. Thyroid. 2016;26(3):434-40.

40. Zheng G, Zhang H, Hao S, Liu C, Xu J. Patterns and clinical significance of cervical lymph node metastasis in papillary thyroid cancer patients with Delphian lymph node metastasis[J]. Oncotarget. 2017;8(34):57089-98.

41. Xiang $D$, Xie $L, X u Y$, et al. Papillary thyroid microcarcinomas located at the middle part of the middle thyroid of the thyroid gland correlates with the presence of neck metastasis[J]. Surgery. 2015;157(3):526-33. DOI:10.1016/j.surg.2014.10.020. 